programme for surgical trainees to enhance patient safety in preventing surgical infection. J Contin Educ Health Prof 2010;30: 257-259.

5. McHugh SM, Corrigan MA, Dimitrov BD, et al. Preventing infection in general surgery: improvements through education of surgeons by surgeons. $J$ Hosp Infect 2011;78:312-316.

6. McHugh SM, Corrigan MA, Dimitrov BD, et al. Role of patient awareness prevention of peripheral vascular catheter-related bloodstream infection. Infect Control Hosp Epidemiol 2011;32: 95-96.

\section{Reply to Weber and Rutala}

To the Editor-We read with interest the commentary by Weber and Rutala. ${ }^{1}$ The authors discuss the contribution of contaminated environmental surfaces within hospitals to transmitting clinically relevant microorganisms frequently associated with healthcare infections. We welcome commentaries of this nature, since the potential for contaminated environmental surfaces to contribute to the chain of healthcare-associated infections is often overlooked and underresearched in favor of more well-publicized and documented mechanisms, primarily the contaminated hands of healthcare workers.

Self-disinfecting surfaces have been the focus of several well-conducted research studies, including studies within our research group. ${ }^{2}$ In the recent study by Karpanen et al, ${ }^{2}$ several frequently touched items-including door handles, grab rails, and toilet seats-in a hospital ward were replaced with equivalent items produced from copper alloy and sampled for microorganisms. The study demonstrated a significant reduction in the microbial bioload on copper as compared with noncopper equivalents.

As Weber and Rutala ${ }^{1}$ state in their commentary, there are limitations to the introduction of copper items in healthcare facilities; however, this is often the case for implementing many novel strategies in infection control, and one should also consider the potential benefits. The data from the study of Karpanen et $\mathrm{al}^{2}$ clearly highlight the value of self-disinfecting surfaces in reducing microbial bioloads from clinical surfaces, and proactive infection control teams may wish to give consideration to where copper surfaces may be of benefit in their respective institutions, for example, in intensive care units or areas with high numbers of immunocompromised patients. An obvious issue is the cost of purchasing and implementing copper items. To our knowledge, cost-effectiveness studies have to date not been undertaken, and investigations to address the added value of copper are therefore clearly warranted, particularly in view of the considerable costs associated with healthcare infections. ${ }^{3}$ The alternative option is perhaps the inertia approach, allowing current environmental surfaces (eg, stainless steel, plastic) to remain dominant in clinical settings. However, these surfaces do not possess any antimicrobial activity and do not contribute to breaking the chain of infection; this is not desirable, since we aspire to design out infection in the modern world.

Copper is a highly efficacious antimicrobial and, as Weber and Rutala ${ }^{1}$ state, has been effective in eliminating important healthcare-associated pathogens. In the United Kingdom, Clostridium difficile is currently one of the leading causes of healthcare-associated infections, and while the number of cases has declined over recent years, it is still a significant cause of morbidity and mortality. Weber and Rutala ${ }^{1}$ comment that copper has not been shown in vitro to kill dormant spores of $C$. difficile. However, in the study by Weaver et al, ${ }^{4}$ copper was shown to kill dormant spores of $C$. difficile within 24-48 hours. Research undertaken by our group has also shown that copper surfaces are highly effective in reducing the viable bioload of $C$. difficile vegetative cells (NCTC 11204 and ribotype 027 ) by approximately 6 logs in both clean and soiled conditions within 30 minutes at room temperature, thus limiting the time for $C$. difficile to develop hardy, resistant environmental spores. ${ }^{5,6}$ Furthermore, studies have also shown that germinating spores of bacteria are more susceptible to killing by disinfectants than dormant spores. At Aston University, we have developed a patented germination solution for $C$. difficile that renders spores susceptible to killing by common biocides, including copper. In our research, we have demonstrated that dormant spores become metabolically active and susceptible to copper surfaces in a short time period following exposure to a $C$. difficile-specific, nontoxic germination solution. Indeed, $99 \%-99.9 \%$ of germinantexposed spores (NCTC 11204 and ribotype 027) are eliminated on both clean and soiled copper surfaces within 3 hours.

Weber and Rutala ${ }^{l}$ are correct when they state that it is impractical or impossible to coat all environmental surfaces and medical devices that could be potentially contaminated with copper. However, a pragmatic approach may be to consider designated areas that may benefit from a background level of antimicrobial protection from copper surfaces supplemented with routine infection control procedures and perhaps novel germination solutions, as alluded to previously. A previous criticism of copper items has been the aesthetic issue, which may be potentially off-putting for patients, visitors, and staff. However, there are now in excess of 300 different antimicrobial copper alloys that span a wide range of colors and surface textures; these may be implemented into clinical settings without disrupting the overall appearance of the environment.

While the antimicrobial activity of copper surfaces has been clearly demonstrated, it is important to appreciate that effective infection control relies on a bundle of measures-some already implemented into clinical practice and some potentially on the horizon-and no single, stand-alone approach is effective at controlling infection. We firmly be- 
lieve, on the basis of our studies and those of others, that self-disinfecting surfaces such as copper are an important additional tool and a significant step forward in helping to reduce the potentially infection-causing microbial bioloads that exist on clinical surfaces. Indeed, we should ask the question, why select a nonantimicrobial surface when we now know that naturally occurring metals have this intrinsic antimicrobial activity?

\section{ACKNOWLEDGMENTS}

Potential conflicts of interest. T.K., A.C., and T.E. report that they have received previous support from the Copper Development Association. All other authors report no conflicts of interest relevant to this article. All authors submitted the ICMJE Form for Disclosure of Potential Conflicts of Interest, and the conflicts that the editors consider relevant to this article are disclosed here.

\section{Tony Worthington, CSci, FIBMS, PhD, FHEA; ${ }^{1}$ Tarja Karpanen, $\mathrm{PhD} ;^{2}$ Anna Casey, $\mathrm{PhD}{ }^{2}$ Peter Lambert, DSc; ${ }^{1}$ Tom Elliott, DSc/FRCpath ${ }^{2}$}

Affiliations: 1. School of Life and Health Sciences, Aston University, Aston Triangle, Birmingham, United Kingdom; 2. Queen Elizabeth Hospital Birmingham, Edgbaston, Birmingham, United Kingdom.

Address correspondence to Tony Worthington, CSci, FIBMS, PhD, FHEA School of Life and Health Sciences, Aston University, Birmingham B4 7ET, United Kingdom (t.worthington@aston.ac.uk).

Infect Control Hosp Epidemiol 2012;33(6):645-646

(C) 2012 by The Society for Healthcare Epidemiology of America. All rights reserved. 0899-823X/2012/3306-0022\$15.00. DOI: 10.1086/665719

\section{REF E REN C ES}

1. Weber DJ, Rutala WA. Self-disinfecting surfaces. Infect Control Hosp Epidemiol 2012;33:10-13.

2. Karpanen TJ, Casey AL, Lambert PA, et al. The antimicrobial efficacy of copper alloy furnishings in the clinical environment: a cross over study. Infect Control Hosp Epidemiol 2012;33:3-9.

3. National Audit Office. Reducing Healthcare Associated Infections in England. London: Stationary Office, 2009.

4. Weaver L, Michels HT, Keevil CW. Survival of Clostridium difficile on copper and steel: futuristic options for hospital hygiene. $J$ Hosp Infect 2008;68:145-151.

5. Wheeldon LJ, Worthington T, Hilton AC, et al. Antimicrobial efficacy of copper surfaces on the spores and vegetative cells of C. difficile: the germination theory. J Antimicrob Chemother 2008; 62:522-525.

6. Wheeldon LJ, Worthington T, Hilton AC, et al. Physical and chemical factors influencing the germination of Clostridium difficile spores. J Appl Microbiol 2008;105:2223-2230.

\section{Failure of HIV Postexposure Prophylaxis after a Work-Related Needlestick Injury}

To the Editor-Transmission of HIV by occupational needlestick injury (NSI) is a rare event, ${ }^{1,2}$ particularly in instances in which a healthcare provider (HCP) receives prompt postexposure prophylaxis (PEP). ${ }^{3}$ We report a case in which PEP failed to prevent HIV transmission.

After placement of a central line in a patient with cryptococcal meningitis for whom HIV was recently diagnosed, a HCP accidentally sustained a NSI to the left thumb with the infiltration needle ( 25 gauge). The exposure site was cleansed thoroughly with soap and water, and the HCP was provided an antiretroviral regimen that consisted of lopinavir/ ritonavir, zidovudine, and lamivudine at the time the NSI was reported (approximately 6 hours after exposure). However, the first doses of antiretroviral medication were not administered until approximately 18 hours after exposure. Serologic tests demonstrated that the source patient had negative results for hepatitis $B$ and $C$, and the results of baseline hepatitis and HIV tests for the exposed HCP were also negative. The source patient had received a diagnosis of HIV 1 day before his arrival, and he had never taken antiretrovirals. He was transferred to another hospital and died less than 24 hours after the accident. No additional blood samples could be recovered for further evaluation of the source patient. The exposed HCP reported no high-risk sexual exposure, no intravenous drug use, and not having undergone HIV testing before. On the 10th day following the exposure, the HCP developed diarrhea (5-6 loose stools per day) without nausea or vomiting, which was considered an adverse effect of the PEP regimen. The regimen was changed to tenofovir (TDF), emtricitabine (FTC), and atazanavir, which the HCP continued to receive without experiencing any further adverse effects. Ultimately, the HCP received a total of 4 weeks of PEP. During the PEP period, the exposed HCP reported strict adherence to both regimens, missing none of the doses. His reports of adherence correlated with a controlled weekly pill count. On the 25th day after exposure, the HCP remained asymptomatic and the results of a second ELISA test for HIV were negative. Approximately 60 days after exposure, the HCP developed a dengue-like illness characterized by fever, thrombocytopenia, muscle pain, and fatigue; the physical examination at that time did not note either adenopathy or rash. Antibody tests for dengue had negative results; however, an ELISA for HIV had positive results (67 days after exposure). The HCP's symptoms subsequently disappeared with symptomatic treatment. On day 74 after exposure, a second ELISA test for HIV had positive results and the results of a Western blot assay were indeterminate. At that time an HIV viral load test was ordered, which detected 60,770 copies $/ \mathrm{mL}$ with a lymphocyte TCD4 count of 672 cells $/ \mathrm{mL}$. The HCP reported that from the time of the NSI until the positive HIV test results, he had no sexual contact or other risk factors for HIV infection.

A blood sample for a genotype assay was collected on the 85th day after exposure (57 days after the last dose of PEP antiretrovirals was administered). Resistance mutations sequenced (ViroSeq) for protease were V3I, E35D, S37D, Q61E, L63P, I64V, C67S, H69Y, and V77I, and for reverse transcrip- 